\title{
HIV vaccine: the best long-term hope
}

An effective vaccine is the best long-term hope for preventing AIDS, says Barry R Bloom from the Albert Einstein College of Medicine, New York, US.

While the new drug 'cocktails' are proving effective in the treatment of AIDS, many people will not have access to these agents because of cost, he notes.*

While Mr Bloom describes the hurdles that must be overcome in developing an HIV vaccine as 'daunting', he believes that the key to success lies in gaining a better understanding of the mechanisms of pathogenesis in HIV infection and the mechanisms involved in gaining protection against HIV infection.

Even a partially effective HIV vaccine would be of use in that it would 'save and extend millions of lives and would reduce secondary transmission to offspring and contacts', says $\mathrm{Mr}$ Bloom.

He adds that it would be helpful if criteria were available to help indicate when a vaccine in development might be eligible to enter phase III clinical trials.

In conclusion, $\mathrm{Mr}$ Bloom expresses concern that investment in HIV vaccine development has declined. He emphasises that the current need is for investment into research concerning the pathogenesis of HIV infection and devoted to developing better vaccine candidates.

* See also Views and Reviews section, this issue, p3-5; 800458017

Bloom BR. A perspective on AIDS vaccines. Science 272: 1888-1890, 28 Jun 1996 